676 HOW CAN WE EXPLAIN GENDER DIFFERENCES IN WORK-RELATED UPPER LIMB SYMPTOMS? A CROSSSECTIONAL STUDY WITH IRISH MANUAL THERAPISTS

${ }^{1}$ Birgit A Greiner*, 'Dervla A Hogan, ${ }^{1,2}$ Sheilah Nolan. 'University College Cork, Dep. Epidemiology and Public Health, Cork, Ireland; ${ }^{2}$ Kerry Health and Safety, Killarney, Ireland

\subsection{6/oemed-2018-ICOHabstracts. 779}

Introduction Female workers commonly show higher prevalence of work-related upper limb disorders (WRULD) than males. Explanations include higher vulnerability to physical and psychosocial work risks, differential exposure to risks and higher muscle activity relative to capacity; research is inconclusive. Objectives were to (1) determine gender-specific WRULD prevalence in Irish Physical Therapists, Athletic Therapists and Physiotherapists, (2) test gender differences in work exposures and in exertion that may explain WRULD disparities, (3) compare whether women's musculoskeletal health was more affected by physical and psychosocial exposures.

Methods 347 hospital-based and self-employed Irish Chartered Physiotherapists, Physical and Athletic Therapists (cluster and random sampling), 114 men and 233 women, completed the Nordic Questionnaire (neck, shoulder, elbows, wrist, finger, thumb symptoms), the Copenhagen Psychosocial Questionnaire (demands, tempo, influence, predictability, social support), physical exposures (repetitive movements, postures, Borg exertion scale). Age-adjusted prevalence odds ratios (POR) of WRULS comparing genders, gender-stratified logistic regression with confounder adjustment.

Result $82 \%$ experienced WRULD in at least one body site without significant difference between women (84\%) and men $(80 \%) \quad(\mathrm{POR}=1.3 ; 0.7-2.3)$, with significantly higher prevalence in women for shoulder (56\% vs $35 \%)$ ( $\mathrm{POR}=1.7,1.2-$ $2.7)$ and neck $(58 \%$ vs $44 \%)(\mathrm{POR}=2.3 ; 1.4-3.7)$. Physical exposures and exertion did not differ by gender. Exertion due to repetitive thumb, arm and wrist movements was more strongly associated with neck and shoulder symptoms in women than in men. Women reported significantly higher tempo and emotional demands, there was no difference in quantitative demands, predictability, influence and social support. The magnitude of associations between psychosocial exposures and WRULDs were similar in gender-stratified analyses.

Discussion Women may be more exposed to certain psychosocial risks and may be more vulnerable to physical exertion. Gender-specific risk assessment for the prevention of WRULS is paramount. Prevention for health care workers in handintensive occupations is warranted given the high ULD prevalence in both genders.

\section{WORK PRODUCTIVITY AND ACTIVITY IMPAIRMENT AFTER TOTAL KNEE ARTHROPLASTY: A 6-MONTHS PROSPECTIVE STUDY}

\footnotetext{
${ }^{1,2} \mathrm{TH}$ Hylkema*, ${ }^{3} \mathrm{~F}$ Selzer, ${ }^{1} \mathrm{M}$ Stevens, ${ }^{4} \mathrm{BA}$ Amick, ${ }^{3} \mathrm{JN}$ Katz, ${ }^{2} \mathrm{~S}$ Brouwer. ${ }^{1}$ University of Groningen, University Medical Centre Groningen, Department of Orthopaedics, The Netherlands; ' University of Groningen, University Medical Centre Groningen, Department of Community and Occupational Medicine, The Netherlands; ${ }^{3}$ Harvard University, Brigham and Women's Hospital, Orthopaedic and Arthritis Centre for Outcomes Research (OrACORe), Boston, USA; ${ }^{4}$ Institute for Work and Health, Toronto, Canada and Robert Stempel College of Public Health and Social Work, Miami, USA
}

10.1136/oemed-2018-ICOHabstracts.780
Introduction Total Knee Arthroplasty (TKA) procedures among working-age patients will rapidly increase. A majority of the patients returns to work after TKA surgery, however, if patients are still limited in daily (work) performance is unknown. Therefore, the aim of this study was to examine impairments in work and activities in TKA patients 6 months postoperative. Moreover, to study associations between preoperative characteristics and impairments in work and daily activities at 6 month follow-up.

Methods A prospective cohort study of patients aged $<70$ years undergoing TKA in the USA. Preoperative data on age, gender, educational level, BMI, pain catastrophizing, pain, function, mental health, knee-related quality of life and health-related quality of life were gathered. At 6 month follow-up patients completed the Work Productivity and Activity Impairment (WPAI) questionnaire, a well validated instrument to measure the effect of the TKA on work productivity and daily activities. Uni- and multivariate linear regressions were used to analyse the association between baseline independent variables and WPAI scores at 6 month follow-up. All models were adjusted for age, gender and BMI.

Results Of the 183 patients included (mean age 61, women $62.3 \%), 74$ reported missing work in the past weeks, accounting for $3.4 \%$ of their working time (absenteeism). On average $20.3 \%$ of their working time was impaired due to problems of their TKA (presenteeism). In addition, 22.8\% of the patients' regular daily activities were impaired due to their TKA (activity impairment). A higher level of pain catastrophizing preoperatively was found as a predictor for presenteeism (Beta 0.35, p<0.001) and activity impairment (Beta 0.47, $\mathrm{p}<0.001)$.

Discussion TKA patients are not often absent from work, but do report impairments in daily functioning, including work. Future interventions should focus on TKA patients with high levels of pain catastrophizing preoperatively.

\section{RELIABILITY, AGREEMENT AND RESPONSIVENESS OF PRODUCTIVITY LOSS (IPCQ-VR) AND HEALTHCARE UTILISATION (TICP-VR) QUESTIONNAIRES FOR SICK WORKERS WITH CHRONIC MUSCULOSKELETAL PAIN}

\footnotetext{
1,2,3Timo T Beemster* ${ }^{*}, 3$ Judith M van Velzen, ${ }^{2,3}$ Coen AM van Bennekom, ${ }^{1}$ Michiel F Reneman, ${ }^{3}$ Monique HW Frings-Dresen. 'University Medical Centre Groningen, Groningen, Department of Rehabilitation Medicine, University of Groningen, The Netherlands; ' Department of Research and Development, Heliomare Rehabilitation Centre, Wijk aan Zee, The Netherlands; ${ }^{3}$ Academic Medical Centre, University of Amsterdam, Department: Coronel Institute of Occupational Health, Amsterdam Public Health research institute, Amsterdam, The Netherlands
}

\subsection{6/oemed-2018-ICOHabstracts.781}

Introduction The aim of this study was to explore test-retest reliability, agreement, and responsiveness of questionnaires on productivity loss (iPCQ-VR) and healthcare utilisation (TiCPVR) for sick workers with chronic musculoskeletal pain who were referred to vocational rehabilitation.

Methods Test-retest reliability and agreement was assessed with a two-week interval. Responsiveness was assessed at discharge after a 15 week vocational rehabilitation (VR) program. Data was obtained from six VR centres in the Netherlands. Testretest reliability was calculateed with intraclass correlation coefficient (ICC) and Cohen's kappa. Agreement was calculated by Standard Error of Measurement, Smallest detectable 
changes (on group and individual level), and percentage observed, positive and negative agreement. Responsiveness was calculated with area under the curve (AUC) obtained from receiver operation characteristic (ROC).

Results A sample of 52 participants on test-retest reliability and agreement and a sample of 223 on responsiveness were included in the study. Of the iPCQ-VR, ICCs ranged from 0.52 to 0.90 , kappa ranged from 0.42 to 0.96 , and AUC ranged from $0.55-0.86$. The ICC of total healthcare utilisation of the TiCP-VR was 0.81 and kappa values of the single healthcare utilisation items ranged from 0.11 to 1 .

Discussion The iPCQ-VR showed good clinimetric properties on working status, number of hours working per week and long term sick leave, and low measurement properties on short term sick leave and presenteeism. The TiCP-VR showed adequate reliability on all healthcare utilisation items together and medication use, but showed low clinimetric properties on the single healthcare utilisation items.

\section{PROGNOSTIC FACTORS FOR WORK PARTICIPATION IN PATIENTS WITH SCIATICA: A SYSTEMATIC REVIEW}

\begin{abstract}
${ }^{1}$ Teddy Oosterhuis, ${ }^{1}$ Veerle R Smaardijk, ${ }^{1}$ PPaul FM Kuijer, ${ }^{2}$ Miranda W Langendam, ${ }^{1}$ Monique HW Frings-Dresen, ${ }^{1}$ Jan L Hoving ${ }^{*}$. 'Academic Medical Centre, Department: Coronel Institute of Occupational Health, Amsterdam Public Health research institute, Amsterdam, The Netherlands; ${ }^{2}$ Academic Medical Centre, University of Amsterdam, Department of Clinical Epidemiology, Biostatistics and Bioinformatics, Amsterdam Public Health research institute, Amsterdam, The Netherlands
\end{abstract}

\subsection{6/oemed-2018-ICOHabstracts.782}

Introduction Sciatica is usually self-limiting with pain and disability decreasing over time, but not all patients fully recover. Prognostic evidence could assist clinicians to better define high risk groups and inform both clinicians and patients with regard to counselling and treatment choices to promote return to work. The objective of this study was to review and summarise prognostic factors of work participation in patients with sciatica.

Methods We searched MEDLINE, CINAHL, EMBASE and PsycINFO till May 2016. Cohort studies, using a measure of work participation as outcome, were included. We used the QUIPS tool for risk of bias assessment and GRADE to rate the quality of the evidence.

Results Based on five studies describing four cohorts $(n=983$ patients) that assessed 19 potential prognostic factors, favourable factors for return to work at long term-term follow up (up to 10 years) included: younger age, better general health, less low back pain or sciatica bothersomeness, better physical function, positive SLR-test, a physician expecting surgery to be beneficial, better pain coping, less depression and mental stress, low physical work load. Study results could not be pooled. Using GRADE, the quality of the evidence ranged from moderate to very low.

Discussion Five studies describing four cohorts identified a wide range of factors: general health, pain and disability, psychological factors and work related factors. Although the number of studies was low and the quality of evidence ranged from moderate to very low, prognostic (modifiable) factors may be used to assist clinicians and occupational healthcare professionals in guiding high risk patients and consider referral for additional care or vocational rehabilitation, or in managing patients' expectations regarding return to work.

\section{4 \\ VALIDATION OF A CONCEPTUAL MODEL FOR SHOULDER PAIN RISK FACTORS IN THREE INDEPENDENT FRENCH WORKING POPULATIONS}

1J Bodin* ${ }^{2,3} \mathrm{R}$ Garlantézec, ${ }^{2} \mathrm{~N}$ Costet, ${ }^{4,5,6} \mathrm{~A}$ Descatha, ${ }^{2,3} \mathrm{JF}$ Viel, ${ }^{1} \mathrm{Y}$ Roquelaure. ${ }^{1}$ INSERM, U1085, IRSET, ESTER Team, University of Angers, Angers, France; ${ }^{2}$ INSERM, U1085, IRSET, 3ERD Team, University Rennes I, Rennes, France; ${ }^{3} \mathrm{CHU}$ Rennes, Rennes, France; ${ }^{4} \mathrm{AP}$-HP UVSQ, Occupational Health Unit, Poincaré University Hospital, Garches, France; ${ }^{5}$ Versailles St-Quentin Univ - Paris Saclay Univ, UMS 011, UMR-S 1168, France; ' INSERM, U1168 UMS 011, Villejuif, France

\subsection{6/oemed-2018-ICOHabstracts.783}

Introduction The aims were to explore the direct and indirect relationships between workplace risk factors, perceived stress and shoulder pain in a sample of male workers from various companies of the industrial sector and to validate the results in workers from two large companies located in France.

Methods Three working populations were analysed: a surveillance network based on a longitudinal design (Cosali) and two samples from companies based on a cross-sectional design. All workers completed a self-administered questionnaire about musculoskeletal symptoms, individual factors and exposure to work constraints. A conceptual model was defined drawing from the literature and the expertise of the authors. Structural equation modelling was used to test the direct and indirect relationships among the variables.

Result Results obtained in the two companies were in majority consistent with those observed in Cosali. In the three samples, industrial constraints influenced physical factors and psychosocial factors whereas market constraints influenced only psychosocial factors. Psychosocial factors and physical factors were correlated and physical factors increased shoulder pain. Shoulder pain was influenced directly by perceived stress only in one sample.

Discussion The results provide a better comprehension of the complexity of the distal and proximal determinants of shoulder pain and highlight that workplace interventions should act on multiple dimensions (i.e. organisational, psychosocial and physical factors) to be more effective.

\section{EFFECTS OF PARTICIPATORY ERGONOMIC INTERVENTION PROGRAM (PEIP) ON MUSCULOSKELETAL AND HEALTH OUTCOMES AMONG HOSPITAL ORDERLIES}

${ }^{1}$ Withaya Chanchai, ${ }^{2}$ Wattasit Siriwong, ${ }^{3}$ Wanpen Songkham, ${ }^{4}$ Pranom Ketsomporn, ${ }^{4}$ Punnarat Sappakitchanchai. ' College of Alied Health Sciences, Sunandha Rajabhat University, Bangkok 10300, Thailand; ' ${ }^{2}$ College of Public Health Sciences, Chulalongkorn University, Bangkok 10330, Thailand; ${ }^{3}$ Faculty of Nursing, Chiang Mai University, Chiang Mai 50200, Thailand; ${ }^{4}$ Department of Patient transfer service, Faculty of Medicine Siriraj Hospital, Mahidol University, Bangkok 10700, Thailand

\subsection{6/oemed-2018-ICOHabstracts.784}

Introduction The participatory ergonomic (PE) approach has been widely used to improve the health outcomes. The purpose of this study was to assess the effects of the participatory ergonomic intervention program (PEIP) can reduce musculoskeletal and health outcomes among hospital orderlies.

Method The randomised controlled trial (RCT) was conducted at tertiary care hospitals during July to December 2014. There were 100 orderlies participated in this study. Data collection was carried out by self-reported questionnaire and Quick exposure check (QEC) technique. 